\title{
Gastric myoelectrical and autonomic cardiac reactivity to laboratory stressors
}

PETER J. GIANAROS, ${ }^{\mathrm{a}}$ KAREN S. QUIGLEY, ${ }^{\mathrm{b}}$ J. TOBY MORDKOFF, ${ }^{\mathrm{b}}$ AND ROBERT M. STERN ${ }^{\mathrm{b}}$

${ }^{a}$ Cardiovascular Behavioral Medicine Research Program, The University of Pittsburgh, Pittsburgh, USA

${ }^{\mathrm{b}}$ Department of Psychology, The Pennsylvania State University, University Park, USA

\begin{abstract}
We evaluated the effects of two laboratory stressors (speech preparation and isometric handgrip) on gastric myoelectrical and autonomic cardiac activity, and the extent to which autonomic responses to these stressors and somatization predict reports of motion sickness during exposure to a rotating optokinetic drum. Both stressors prompted a decrease in preejection period (PEP) and respiratory sinus arrhythmia (RSA), and an increase in a dysrhythmic pattern of gastric myoelectrical activity, termed gastric tachyarrhythmia. Stressor-induced decreases in RSA and higher somatization scores predicted increased reports of motion sickness during drum rotation. These results demonstrate that laboratory stressors concurrently affect gastric myoelectrical activity and autonomic control of the heart, and that stressor-induced decreases in RSA and higher levels of somatization predict motion sickness susceptibility.
\end{abstract}

Descriptors: Electrogastrography, Impedance cardiography, Motion sickness, Parasympathetic, Somatization, Stress, Sympathetic

Individuals often report epigastric symptoms during stressful experiences (Stern \& Higgins, 1969). These symptoms are reflected in such expressions as "a nervous stomach" and may range in severity from a simple awareness of gastric activity to a sensation that disrupts ongoing behavior. Although recent epidemiological findings indicate that there is an increased prevalence of gastrointestinal symptoms among individuals with affective disorders (Hochstrasser \& Angst, 1996; Lydiard et al., 1994; Marten et al., 1993) and an increased prevalence of negative affect and stressful life events among individuals with functional gastrointestinal disorders (Bennett et al., 1997; Bennett, Tennant, Piesse, Badcock, \& Kellow, 1998; Whitehead, 1992, 1996; Whitehead, Crowell, Robinson, Heller, \& Schuster, 1992), the effects of stressful experiences on the human stomach have been studied infrequently over the last several decades. One reason for the relative dearth of research on gastric reactivity to stress is that most gastrointestinal measurement procedures are invasive.

This research was supported by a National Research Service Award (1F31MH12418-01) from the National Institute of Mental Health to Peter J. Gianaros. We thank David Lozano and Marty Gillman for their technical assistance and for providing analysis software, Robert Andelman, Shannon Rushing, and Jessica Neff for their help in data acquisition, and Gary G. Berntson for his helpful suggestions regarding autonomic space analyses. We also thank Lorenz van Doornen, Natasha Tokowicz, and two anonymous reviewers for their helpful comments on an earlier version of this manuscript.

A preliminary report of these findings was made at the 40th Annual Meeting of the Society for Psychophysiological Research, October 18-22, 2000, San Diego, CA.

Address reprint requests to: Peter J. Gianaros, E1329 Western Psychiatric Institute and Clinic, University of Pittsburgh, 3811 O'Hara St., Pittsburgh, PA 15213, USA. E-mail: pjg4@ pitt.edu.
For example, the earliest work to document the effects of stress and negative affect on gastric activity was conducted with individuals whose stomachs were exposed with a fistula (e.g., Beaumont, 1833; Wolf \& Wolff, 1947). More recently, gastric intubation and intraluminal pressure recording techniques have been used to show that stressors such as electric shock (Camilleri, Malagelada, Kao, \& Zinsmeister, 1984, 1986), challenging cognitive problems (Holtman, Singer, Kriebel, Stäcker, \& Goebell, 1989), irrigation of the tympanic membrane, and cold pressor stimuli (Thompson, Richelson, \& Malagelada, 1982a, 1982b, 1983) delay gastric emptying and decrease gastric motility. Two limitations of these studies, however, are that both subjective distress and gastrointestinal responses that are independent of experimental manipulations may be elicited by the invasive procedures employed (Stern, 1983). To date, few studies have used electrogastrography (EGG) to study the effects of laboratory stressors on gastric myoelectrical activity. The advantages of EGG over other gastrointestinal procedures are that (a) noninvasive EGG recording does not interfere with the activity of the stomach, and (b) specific patterns of gastric myoelectrical activity are associated with changes in autonomic activity and the report of physical symptoms, such as nausea (Stern, Koch, \& Muth, 2000).

In humans, normal gastric myoelectrical activity occurs at a frequency of approximately 3 cycles per minute (cpm). The frequency of gastric myoelectrical activity is identical to the frequency of stomach contractions when they occur, and the amplitude of normal 3-cpm activity is correlated positively with the contractile activity of the stomach (Stern et al., 2000). Both normal 3-cpm activity and, consequently, the contractile activity of the stomach may be disrupted, however, by two types of erratic or dysrhythmic gastric myoelectrical activity: gastric bradyarrhythmia (1-2 cpm) 
and gastric tachyarrhythmia (4-9 cpm; Koch, 1993). Whereas gastric bradyarrhythmia has not been reliably associated with a specific behavioral state or clinical outcome, gastric tachyarrhythmia has been associated with the experience of nausea during motion sickness (e.g., Stern et al., 1985; Stern, Koch, Stewart, \& Lindbald, 1987), gastroparesis (e.g., Liberski, Koch, Atnip, \& Stern, 1990), pregnancy (e.g., Koch, Stern, Vasey, \& Dwyer, 1990), functional dyspepsia (e.g., Koch, 1998), and gastric ulceration (e.g., Geldof et al., 1989). Furthermore, several studies have shown that the expression of gastric tachyarrhythmia during the experience of nausea is associated with an increase in sympathetic and decrease in parasympathetic activation (Koch \& Stern, 1996; Stern $\&$ Koch, 1996). Prior research, for example, has shown that motion sickness-induced gastric tachyarrhythmia is associated with decreased parasympathetic cardiac activity (Hu, Grant, Stern, \& Koch, 1991; Uijtdehaage, Stern, \& Koch, 1992, 1993), increased skin conductance (Hu et al., 1991; Miller, Sharkey, Graham, \& McCauley, 1993), and increased plasma catecholamine levels (Koch, Stern, Vasey, Seaton et al., 1990). Prior experiments investigating the effects of laboratory stressors on gastric myoelectrical activity have shown that 3-cpm activity decreases in response to the cold pressor test (Riezzo, Porcelli, Guerra, \& Giorgio, 1996; Stern, Vasey, Hu, \& Koch, 1991) and viewing aversive films (e.g., limb mutilation; Stewart, 1987), and that 3-cpm activity decreases and gastric tachyarrhythmia increases during shock avoidance (Muth, Koch, Stern, \& Thayer, 1999). Neither sympathetic nor parasympathetic activity, however, was evaluated in these experiments. Thus, it is not possible to evaluate whether these stressor-induced changes in gastric myoelectrical activity corresponded to particular patterns of autonomic activity.

In the present study, we evaluated whether two laboratory stressors that elicit a reciprocal increase in sympathetic and decrease in parasympathetic cardiac activity also elicit a decrease in 3 -cpm activity and increase in gastric tachyarrhythmia. We employed a psychological stressor (speech preparation) and a physical stressor (sustained isometric handgrip), which have been shown previously to increase sympathetic and decrease parasympathetic cardiac activity (Berntson et al., 1994; Cacioppo et al., 1994; Kino, Lance, Shahamatpour, \& Spodick, 1975; Pollack \& Obrist, 1988; Saab, Matthews, Stoney, \& McDonald, 1989). ${ }^{1}$ For each task, subjective reports of nausea, measures of 3-cpm activity and gastric tachyarrhythmia, and noninvasive estimates of sympathetic (preejection period; PEP) and parasympathetic (respiratory sinus arrhythmia; RSA) cardiac activity were obtained. Lower values of PEP reflect greater myocardial contractility due to increased sympathetic (specifically $\beta$-adrenergic) outflow to the myocardium under conditions of little change in preload or afterload (Cacioppo et al., 1994; Lewis, Leighton, Forester, \& Weissler, 1974; Sherwood et al., 1990). Higher values of RSA indicate a greater parasympathetically mediated variation in heart period that coincides with inspiration and expiration after controlling for significant respiratory adjustments (Berntson, Cacioppo, \& Quigley, 1993b; Berntson et al., 1997; Grossman, Karemaker, Wieling, 1991; Porges \& Bohrer, 1990). If the speech preparation and isometric handgrip tasks elicit a decrease in PEP and RSA, then we

${ }^{1}$ In addition to eliciting reciprocal changes in autonomic activity, these tasks were chosen because they yielded very few movement artifacts in the electrogastrogram. In prior pilot work, we employed laboratory stressors (e.g., speech delivery, mental arithmetic, etc.) that required vocalization or repeated movements; however, these tasks elicited a substantial number of EGG artifacts. would also expect to observe a decrease in 3-cpm activity and an increase in gastric tachyarrhythmia. Such findings from each task would parallel prior motion sickness research and would provide converging evidence that this pattern of gastric myoelectrical reactivity is associated with a reciprocal increase in sympathetic and decrease in parasympathetic autonomic activation as measured at the level of the heart.

A secondary aim of the present study was to determine whether individual differences in motion sickness susceptibility could be predicted by autonomic cardiac reactivity to the laboratory stressors and trait levels of somatization. Prior research suggests that susceptibility to motion sickness may be predicted by both autonomic (e.g., Cowings, Suter, Toscano, Kamiya, \& Naifeh, 1986; Kohl \& Homick, 1983; Money, Lackner, \& Cheung, 1996; Parker, 1971; Stern \& Koch, 1996) and personality (e.g., Wilding \& Meddis, 1972) variables. For example, Parker demonstrated that skin conductance responses to a film, which depicted a car traveling along a circuitous road, predicted later reports of sea sickness elicited by a sail boat. Uijtdehaage et al. (1992) also demonstrated that increases in RSA following meal ingestion correlated negatively with motion sickness symptoms that were later induced by a rotating optokinetic drum. In that study, meal-induced increases in RSA also correlated positively with 3-cpm gastric myoelectrical activity and negatively with gastric tachyarrhythmia elicited by drum rotation. These results suggested that changes in RSA may be used to predict both behavioral and gastric myoelectrical responses to vection-induced motion sickness. It is currently unknown, however, whether environmental challenges that elicit other patterns of autonomic response (e.g., decreased parasympathetic cardiac activity) may be used to predict behavioral or physiological responses to a nauseogenic stimulus. According to a recent model of motion sickness susceptibility proposed by Stern and Koch (1996), reciprocal changes in autonomic drive (i.e., increased sympathetic and decreased parasympathetic activation) during stress may indeed predict one's susceptibility to nausea or motion sickness. In the present study, we tested this prediction by exposing participants to a rotating optokinetic drum following the completion of the speech preparation and isometric handgrip tasks. If reciprocal changes in autonomic activity during stress predict motion sickness susceptibility, then concurrent decreases in RSA and PEP during the speech preparation and isometric handgrip tasks should predict greater reports of motion sickness during vection. The extent to which changes in RSA and PEP to the laboratory stressors predict changes in normal 3-cpm activity and gastric tachyarrhythmia during exposure to the rotating optokinetic drum was also examined.

In addition to autonomic responses, neuroticism has been used frequently to predict motion sickness susceptibility. It is thought that higher levels of neuroticism relate to increased motion sickness susceptibility; however, results in this area are mixed (for review, see Kennedy, Dunlap, \& Fowlkes, 1990). A limitation of the research on motion sickness susceptibility and neuroticism is that few investigators have provided a hypothesis that would explain a relationship between the two constructs. One possible explanation is that heightened attention to physical symptoms may be related to reports of motion sickness. Kennedy et al., for instance, recently suggested that "being concerned with bodily complaints may encourage one to report [motion sickness] symptoms" (p. 183). An individual's tendency to report distress from physical symptoms is referred to as somatization (Derogatis, Rickels, \& Rock, 1976). Strong relationships between somatization and neuroticism have been documented in a number of clinical and non-clinical populations; however, neuroticism scores may not 
necessarily reflect an individual's level of somatization (Costa \& McCrae, 1987; Watson \& Pennebaker, 1989). Thus, whereas neuroticism represents a broad personality trait, somatization is a narrower construct relating specifically to the tendency to report distress from physical symptoms, which may be a more relevant predictor of motion sickness symptomatology. The fact that somatization and neuroticism are partially correlated could explain prior mixed results when the broader construct, neuroticism, is used as a motion sickness predictor. In the present study, we hypothesized that greater levels of somatization would be related to increased reports of vection-induced motion sickness.

\section{Method}

\section{Participants}

Participants were 59 undergraduate students ( 34 women) aged 18 to 34 years, who were recruited from psychology courses at The Pennsylvania State University. Participants received extra credit for participation and all procedures were approved by the Institutional Review Board of The Pennsylvania State University. Individuals with (a) cardiovascular, gastrointestinal, or respiratory illness, (b) a body mass $35 \%$ above or below normal body weight, (c) an exercise routine exceeding 20 hours per week, or (d) basal blood pressure greater than $150 \mathrm{mmHg}$ systolic or $90 \mathrm{mmHg}$ diastolic were excluded from participation. The mean body surface area $\left(\right.$ BSA in meters ${ }^{2}=$ weight $(\mathrm{kg})^{0.425} \times$ height $\left.(\mathrm{cm})^{0.725} \times .007184\right)$ was 1.68 for women and 1.98 for men.

\section{Psychophysiological Measures}

Electrogastrography. Three disposable $\mathrm{Ag} / \mathrm{AgCl}$ electrodes were used to record the EGG signal. Active electrodes were positioned (a) on the abdominal midline, just above the umbilicus and (b) approximately $6 \mathrm{~cm}$ to the left and $3 \mathrm{~cm}$ superior to the midline electrode. A reference electrode was positioned approximately $10 \mathrm{~cm}$ to the right of the midline and $3 \mathrm{~cm}$ above the umbilicus. Field effect transistor electrodes (Fetrodes; UFI Corporation, Morro Bay, CA) were attached to the active EGG leads to increase the signal-to-noise ratio. The EGG signal was passed through a UFI Fetrode bioamplifier (Model 2121FT) before being sent to a Gould recorder (Model 3000, Cleveland, $\mathrm{OH}$ ), which was equipped with a modified Universal coupler (bandwidth: 0.008 to $0.30 \mathrm{~Hz}$ ). The EGG signal was digitized at $4.267 \mathrm{~Hz}$ using a 12 bit A/D converter and was stored for off-line processing using Labtech Notebook 6.3 (Laboratory Technologies, Wilmington, MA).

Spectral analyses using fast Fourier transforms (FFTs) were performed on 5-min epochs of EGG data from all baseline and experimental epochs. Prior to spectral analysis, the EGG time series for each 5-min epoch was linearly detrended and meancentered. A Hamming window was then used to taper the EGG signal. After windowing, spectral density estimates were derived from FFTs in 0.25 -cpm-wide bins within the frequency range of $2.5-15 \mathrm{cpm}$. The percentage of total power was calculated for the $3 \mathrm{cpm}(2.5-3.75 \mathrm{cpm})$ and gastric tachyarrhythmic $(4-9.75 \mathrm{cpm})$ bandwidths using the following equations: \% 3-cpm activity = (2.5-3.75 cpm power $/ 2.5-15 \mathrm{cpm}$ power $) \times 100$; \% gastric tachyarrhythmia $=(4-9.75 \mathrm{cpm}$ power $/ 2.5-15 \mathrm{cpm}$ power $) \times 100$ (Uijtdehaage et al., 1992).

Electrocardiography and impedance cardiography. Electrocardiogram (ECG), impedance cardiogram, and impedance pneumogram signals were obtained using a Minnesota Impedance Cardiograph (Model 304 B). ECG signals were obtained from two disposable $\mathrm{Ag} / \mathrm{AgCl}$ electrodes that were positioned in a modified Lead II configuration. Aluminum-coated, Mylar band electrodes (Instrumentation for Medicine, Greenwich, CT) were used to record basal thoracic impedance $\left(Z_{0}\right)$, the first derivative of the pulsatile change in thoracic impedance $(\mathrm{dZ} / \mathrm{dt})$, and the integrated $\mathrm{dZ} / \mathrm{dt}$ signal $\left(\Delta Z_{d}\right)$. Voltage electrodes were placed circumferentially around the base of the neck and around the thorax over the xiphisternal junction. Current electrodes were attached at least $3 \mathrm{~cm}$ above (neck) or below (chest) the voltage electrodes and passed a 4-mA 100-kHz AC current. ECG and impedance cardiogram signals were digitized at $1000 \mathrm{~Hz}$ using a 12-bit A/D board. Prior to analysis, the ECG and $\mathrm{dZ} / \mathrm{dt}$ signals were decimated to $500 \mathrm{~Hz} ; Z_{0}$ and $\Delta Z_{d}$ (impedance pneumogram) signals were decimated to $250 \mathrm{~Hz}$. All impedance- and electrocardiogram-derived waveforms $\left(Z_{0}, \mathrm{dZ} / \mathrm{dt}, \Delta Z_{d}, \mathrm{ECG}\right)$ were acquired and processed off-line using a customized software package (ANS Suite 6.1, The Ohio State University, Columbus, $\mathrm{OH}$ ).

Heart period was derived from the ECG signal as the interval in milliseconds between sequential $\mathrm{R}$ spikes. Interbeat intervals were examined and edited for artifacts using the artifact detection procedure developed by Berntson, Quigley, Jang, and Boysen (1990). Less than $1 \%$ of the heart period data contained artifacts. Average heart periods for each minute of the baseline and experimental epochs were then calculated using a weighted-beat algorithm (Berntson, Cacioppo, \& Quigley, 1995). One-minute ensemble averages of PEP were calculated as the interval in milliseconds between the Q wave of the electrocardiogram and the B-point of the $\mathrm{dZ} / \mathrm{dt}$ waveform.

RSA was used as an estimate of parasympathetic cardiac control and was calculated for each minute of the baseline and experimental periods. Prior to calculation, artifacts (rapid transitions) in the heart period time series were identified and then removed with a smoothing function. The percentage of rapid heart period transitions corrected in the present data set was $0.068 \%$. The weighted heart period time series was then linearly detrended, mean-centered, and tapered using a Hamming window. FFTs of the 60-s heart period time series were then used to calculate the spectral power (in milliseconds squared per hertz) in the respiratory frequency bandwidth $(0.12-0.40 \mathrm{~Hz})$. The natural $\log$ of the spectral power in the respiratory bandwidth was then taken as the estimate of RSA.

Respiration was obtained using the impedance pneumography procedure described by Ernst, Litvack, Lozano, Cacioppo, and Berntson (1999). Specifically, the integrated dZ/dt signal $\left(\Delta Z_{d}\right)$ from impedance cardiographic recordings was used to derive a respiratory signal. The mean respiratory rate and amplitude were then derived from FFTs of the respiratory time series for each minute of the baseline and task periods.

\section{Motion Sickness Induction}

A rotating optokinetic drum was used to elicit motion sickness symptoms (see Muth, Stern, \& Koch, 1998, for complete discussion of the rotating optokinetic drum). The drum is a cylinder $91.5 \mathrm{~cm}$ in height and $76 \mathrm{~cm}$ in diameter. Alternating $3.8 \mathrm{~cm}\left(5.7^{\circ}\right)$ black and $6.2 \mathrm{~cm}\left(9.3^{\circ}\right)$ white vertical stripes line the interior of the drum. The participant was seated in the center of the drum as it rotated about her or him at a rate of $60^{\circ} \mathrm{s}$. During drum rotation, all participants reported vection (i.e., illusory self-motion); 5 participants did not report any symptoms of motion sickness.

\section{Symptom Assessment and Dispositional Measures}

The Nausea Profile (NP; Muth, Stern, Thayer, \& Koch, 1996) was administered to participants after the baseline, task, and drum 
rotation periods. The NP provides a total nausea score as well as subscale scores for the following symptom dimensions that were derived from prior factor analyses: gastrointestinal distress (sick, queasy, ill, stomach awareness/discomfort, vomiting), somatic distress (shaky, lightheaded, sweaty, tired/fatigued, weak, warm), and negative affect (upset, worried, hopeless, panicked, nervous, scared/ afraid). The total NP score was calculated as the percentage of 153 total possible points on the NP; each of the 17 questions had a maximum possible score of 9. Similarly, gastrointestinal distress, somatic distress, and negative affect scores were calculated as the percentage of possible points for each subscale; thus, all NP scores ranged from 0 to $100 \%$. Prior to statistical analyses, all NP scores were square root transformed to correct distributional violations. Subjective symptoms of motion sickness were obtained during drum rotation using the Pensacola Diagnostic Index (PDI; Graybiel, Wood, Miller, \& Cramer, 1968). PDI scores reflect a composite rating of several symptoms of motion sickness (dizziness, headache, warmth, sweating, drowsiness, salivation, and nausea) and range from 0 to 63 .

Somatization was assessed using the somatization subscale of the SCL-90 (Derogatis, 1977). This subscale was administered to each participant during the electrode stabilization period (see below). Somatization scores were obtained from the participant's mean rating of the extent to which she or he had been distressed by the following symptoms in the last month using a 4-point Likerttype scale $(0=$ not at all, $3=$ extremely $)$ : faintness or dizziness, pains in the heart or chest, nausea or upset stomach, trouble breathing, hot or cold spells, numbness, weakness.

\section{Design and Procedure}

All participants were instructed to fast for a minimum of $3 \mathrm{hr}$ prior to the experiment. Upon arrival at the laboratory, the participant was given a verbal briefing on the nature of the study, the recording procedure, and electrode attachment. After informed consent was obtained, the participant completed a health questionnaire to determine eligibility and electrodes were attached. Electrode attachment was followed by a 10-min electrode stabilization period and an 8-min "vanilla baseline" (Jennings, Kamarck, Stewart, Eddy, \& Johnson, 1992; see below). Following the baseline period, participants were exposed to a speech preparation task and an isometric handgrip task in a pseudorandom, counterbalanced order. An 8-min vanilla recovery period followed each task.

Vanilla baseline and recovery. During the baseline and recovery periods, participants performed a neutral color counting task (Jennings et al., 1992) for $8 \mathrm{~min}$. At the beginning of the period, the participant was instructed to count the number of times a specified $8 \times 10 \mathrm{~cm}$ colored rectangle appeared on a computer monitor that was positioned approximately $90 \mathrm{~cm}$ in front of the participant. During the counting task, a different colored rectangle (blue, brown, green, purple, or white) was presented every $15 \mathrm{~s}$. The participant reported how many times she or he observed the designated color at the end of each baseline/recovery period. Feedback about task performance was not provided, and a different color was counted during each vanilla baseline and recovery period.

Speech preparation. A modified version of the speech stressor developed by Saab et al. (1989) was used in the present study. Specifically, each participant was asked to imagine that a roommate had stolen $\$ 300.00$ from her or him. The participant was then given 5 min to prepare a 5 -min speech about what she or he would say to the roommate. The participant was asked to give a welldeveloped speech and was told that the speech would be videotaped and evaluated. A video camera was positioned approximately $90 \mathrm{~cm}$ in front of the participant during the speech preparation period. During the preparation period, participants were informed of the remaining time each minute. After the preparation period, participants were instructed that speech delivery was not required and no actual video recordings were to be made. During the debriefing session following the experiment, participants were asked to refrain from discussing the fact that speech delivery was not required with other people who might later be participants in the study (e.g., friends, classmates, etc.).

Isometric handgrip task. In the isometric handgrip task, the participant was asked to grip a hand dynamometer (Model PL 3030J1, Jamar, Jackson, MS) at $20 \%$ of her or his maximum strength for a 5-min period. Maximum strength was determined after the informed consent procedure. Specifically, each participant was instructed to squeeze the device as hard as she or he could twice for $2 \mathrm{~s}$; each trial was separated by a 10 -s rest period. The mean of the two trials was taken as the participant's maximum force and $20 \%$ of this mean was calculated. During the 5-min task, an experimenter monitored the participant's performance and instructed her or him to squeeze harder if performance fell beneath the $20 \%$ mark. Participants were informed each minute of the remaining time for this task.

Rotating optokinetic drum. Following the recovery period of the second task, the band electrodes were removed and the participant was escorted to another testing room, which housed the rotating optokinetic drum. In this phase of the experiment, the participant was seated within the drum for a 6-min resting baseline. The baseline period was followed by a maximum of $16 \mathrm{~min}$ of drum rotation. During the rotation period, subjective symptoms of motion sickness were obtained using the PDI approximately every $3 \mathrm{~min}$.

\section{Data Quantification}

Prior to statistical analyses, 1-min estimates of heart period, PEP, RSA, and respiratory parameters were averaged for the baseline and task periods. The effects of each stressor (speech preparation, isometric handgrip) on physiological and NP data were evaluated using 2 (Gender) $\times 2$ (Task Order) $\times 3$ (Period: Baseline, Speech Preparation, Handgrip) Multivariate Analyses of Variance (MANOVAs). The Pillai-Bartlett trace was used as the multivariate test statistic. No main effects or interactions involving task order were found for any variable; therefore, this factor was removed prior to subsequent analyses. Physiological variables that were highly correlated $(r \mathrm{~s}>.60)$ were included in the same MANOVA and subsequent repeated measures ANOVAs were performed on each dependent measure with Greenhouse-Geisser (1959) corrections $(\epsilon)$ for inflated degrees of freedom. Main effects of Period were followed with three planned pairwise comparisons between the (a) baseline and speech preparation, (b) baseline and handgrip, and (c) speech preparation and handgrip tasks using Bonferroni-corrected, within-subject $t$ tests. A Type I error rate of .05 was adopted for all statistical analyses. This experimentwise Type I error rate was maintained at .05 for post hoc comparisons involving overall task effects by setting the $\alpha$ of each of the three comparisons to .017 . Degrees of freedom in the statistical analyses varied because of missing data due to movement artifacts in the EGG record $(n=5)$, impedance cardiography acquisition failure $(n=16)$, and experi- 
menter error in the administration of the NP $(n=3)$ during the vanilla baseline, speech preparation, or isometric handgrip tasks.

A hierarchical linear regression analysis (Cohen \& Cohen, 1983) was used to predict participants' motion sickness scores from somatization scores and basal and average task estimates of RSA and PEP. Specifically, participants' motion sickness scores were regressed hierarchically upon two sets of independent variables, which were entered in the following order: Set $1=$ basal levels of RSA and PEP; Set 2 = average task levels of RSA and PEP and somatization scores. We evaluated the proportion of variance in motion sickness scores accounted for by the first set of predictors $\left(R^{2}\right)$, and the increment in the proportion of variance accounted for by the second set of predictors $\left(\Delta R^{2}\right)$. We also examined the partial $(p r)$, semipartial $(s r)$, and zero-order correlation coefficients for each variable. Similar hierarchical regression analyses were used to predict drum rotation levels of the percentages of gastric tachyarrhythmia and 3-cpm activity after partialling drum rotation basal levels of gastric myoelectrical activity in the first step.

\section{Results}

\section{Heart Period}

The effects of speech preparation and the isometric handgrip task on heart period are shown in Figure 1. Heart period differed significantly between the vanilla baseline, speech preparation, and handgrip tasks, Pillai-Bartlett trace $=.704, F(2,40)=47.62, p<$ .001 . The main effect of Gender, $F(1,41)=1.00$, and the Gender $\times$ Period interaction, $F(2,40)=1.01$, did not reach statistical significance. Compared to baseline, heart period decreased significantly during speech preparation, $t(50)=11.23, p<.001$, and the isometric handgrip task, $t(44)=7.28, p<.001$. In addition, heart period was lower during speech preparation compared to the handgrip task, $t(42)=4.09, p<.001$.

\section{Pre-ejection Period and Respiratory Sinus Arrhythmia}

The upper panel of Figure 2 illustrates the task-induced changes in PEP. A main effect of Period, Pillai-Bartlett trace $=.73, F(2,40)=$ 52.91, $p<.001$, on PEP suggested a change in $\beta$-adrenergic drive to the heart across the tasks. Neither the main effect of Gender, $F<1$, nor the Gender $\times$ Period interaction, $F<1$, reached

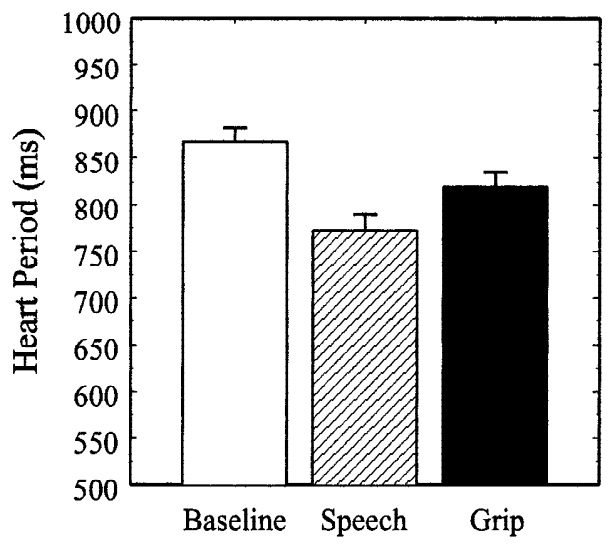

Figure 1. Heart period during the vanilla baseline, speech preparation (Speech), and isometric handgrip (Grip) tasks. Error bars represent the standard error of the mean.
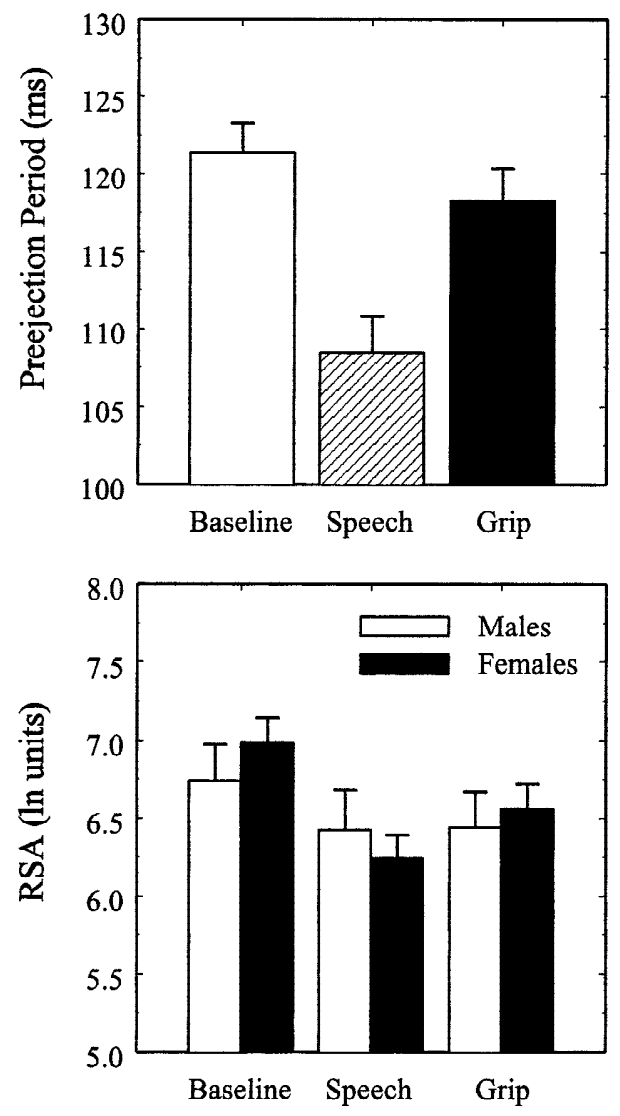

Figure 2. Preejection period (PEP; upper panel) and respiratory sinus arrhythmia (RSA; lower panel) during the vanilla baseline, speech preparation (Speech), and isometric handgrip (Grip) tasks. Error bars represent the standard error of the mean.

statistical significance. Compared to baseline, both speech preparation, $t(50)=11.24, p<.001$, and isometric handgrip, $t(44)=$ $7.44, p<.001$, elicited significant decreases in PEP; however, speech preparation resulted in a greater decrease in PEP than isometric handgrip, $t(42)=6.26, p<.001$.

The lower panel of Figure 2 illustrates RSA levels as a function of the vanilla baseline, speech preparation, and isometric handgrip tasks for males and females. A Gender $\times$ Period interaction, PillaiBartlett trace $=.15, F(2,40)=3.47, p=.04$, modified the main effect of Period, Pillai-Bartlett trace $=.54, F(2,40)=23.12, p<$ .001 , on RSA. The main effect of Gender, however, did not reach statistical significance, $F<1$. Whereas females showed a statistically significant change in RSA across the three time periods, Pillai-Bartlett trace $=.71, F(2,26)=32.20, p<.001$, the main effect of Period on RSA for males was marginal, Pillai-Bartlett trace $=.34, F(2,13)=3.36, p=.07$. Post hoc comparisons indicated that females showed a significant decrease in RSA during both speech preparation, $t(31)=7.63, p<.001$, and isometric handgrip, $t(28)=6.22, p<.001$, compared to baseline. For females, RSA was lower during speech preparation compared to the handgrip task, $t(27)=2.23, p=.03$; however, this difference did not reach the Bonferroni-corrected level of statistical significance. In contrast, males showed a significant decrease in RSA in response to the speech preparation task, $t(18)=3.10, p=.006$, and a marginal decrease in RSA to the isometric handgrip task, $t(15)=$ $2.03, p=.06$; however, no difference in RSA was observed between the handgrip and speech preparation tasks for males, $t<1$. 


\section{Respiratory Parameters}

A MANOVA, which included respiratory rate and amplitude in the same model, revealed a main effect of Period, Pillai-Bartlett trace $=$ $.25, F(4,38)=3.12, p=.03$, and Gender, Pillai-Bartlett trace $=$ $.37, F(2,40)=11.73, p<.001$; however, the Gender $\times$ Period interaction did not reach statistical significance, $F(4,38)=1.91$. Separate repeated measures ANOVAs on respiratory rate and amplitude revealed a main effect of Period on respiratory rate, $F(2,82)=3.29, p=.04, \epsilon=.99$, but not respiratory amplitude, $F<1$. The main effect of Gender in the multivariate analysis was due to a greater respiratory amplitude across all tasks among females compared to males, $F(1,41)=19.44, p<.001$; no main effect for Gender was found for respiratory rate, $F<1$. Gender differences in respiratory amplitude derived from the impedance pneumography procedure likely were due to greater overall levels of thoracic impedance among females, who, on average, are more likely to have smaller thorax volumes than males (Bassett-Frey, Doerr, \& Miles, 1982).

Post hoc comparisons of respiratory rate revealed a significant increase from baseline, $M=15.60$ (breaths per minute; bpm), $S E=.33$, to speech preparation, $M=16.39 \mathrm{bpm}, S E=.35 ; t(50)=$ $3.79, p<.001$, but no differences between baseline and the isometric handgrip task, $M=15.93 \mathrm{bpm}, S E=.38 ; t(44)=1.58$, or between speech preparation and isometric handgrip, $t(42)=1.75$.

Because centrally mediated changes in RSA may be confounded with those reflecting peripheral contributions from taskinduced alterations in respiratory rate (Grossman et al., 1991; Berntson, Cacioppo, \& Quigley, 1993a), within-subject regression analyses were used to derive residualized RSA values using corresponding respiratory rates as predictors. Concordant with the analyses of nonresidualized RSA, a main effect of Period, PillaiBartlett trace $=.355, F(2,40)=14.22, p<.001$, but not Gender, $F<1$, was observed for respiration-corrected RSA. In addition, a marginal Gender $\times$ Period interaction was also obtained, PillaiBartlett trace $=.115, F(2,40)=2.61, p=.08$. Given the consistency of these findings with those of the prior analyses, it is unlikely that changes in respiratory rate substantially altered taskinduced changes in RSA. Therefore, uncorrected RSA values were used in subsequent analyses.

\section{Individual Differences in Autonomic Response}

Figure 3 displays the average autonomic cardiac response elicited by the speech preparation and isometric handgrip tasks using an autonomic space depiction (Berntson, Cacioppo, \& Quigley, 1991, 1993b). The procedures used to derive these figures were adapted from those developed by Berntson, Cacioppo, \& Fieldstone (1996). ${ }^{2}$ At the group level, both tasks appear to have elicited a reciprocal increase in sympathetic and decrease in parasympathetic cardiac control. Within and between the two tasks, however, individuals differed in both the magnitude and pattern of autonomic response

${ }^{2} \mathrm{PEP}$ and RSA responses to the speech preparation and isometric handgrip tasks were first standardized by dividing each change score (e.g., $\triangle \mathrm{PEP}=$ Speech Task PEP - Basal PEP) by the standard deviation of that change score. Heart period responses to each task were then regressed upon the corresponding standardized PEP and RSA responses to determine the appropriate spacing of the abscissa and ordinate of the bivariate autonomic plane. Together, standardized PEP and RSA responses accounted for a moderate amount of the variance in task-induced heart period changes, $R^{2}=.52, F(1,40)=48.77, p<.001$. Because the standardized slopes relating changes in heart period to changes in RSA, $\beta=.485, p<.001$, and PEP, $\beta=.495, p<.001$, were similar, the sympathetic and parasympathetic axis lengths were equated in the bivariate depictions.

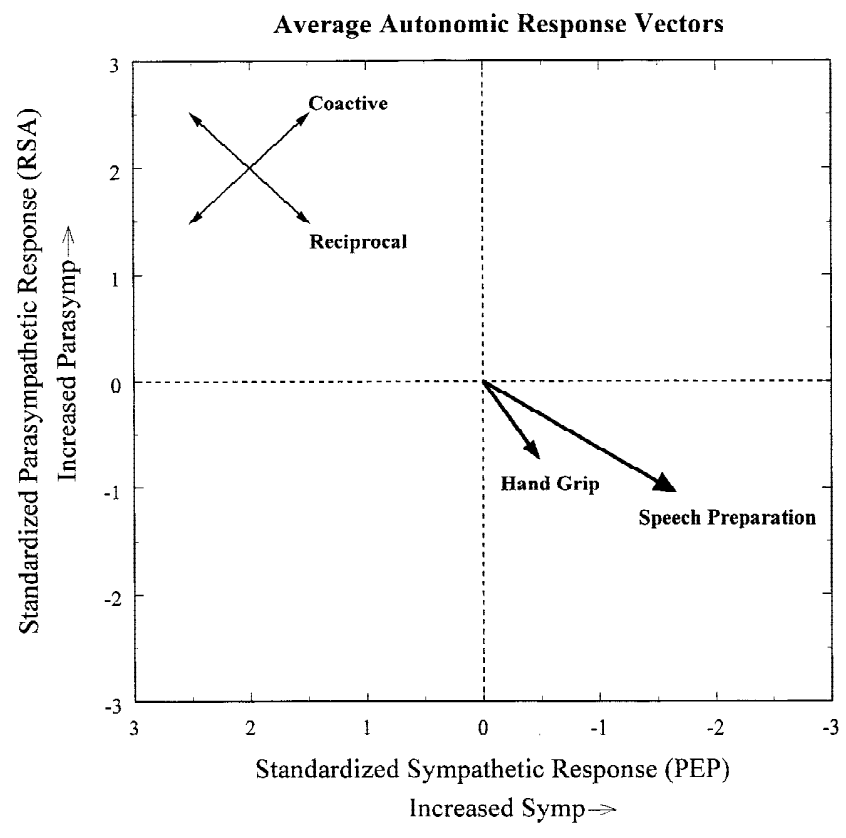

Figure 3. Mean autonomic response vectors representing the standardized change in preejection period (PEP) and respiratory sinus arrhythmia (RSA) from baseline. Standard errors of the mean standardized PEP response are represented by the length of the arrowheads; standard errors of the mean standardized respiratory sinus arrhythmia response are represented by the width of the arrowhead.

(see Figure 4). PEP and RSA did not correlate within the speech preparation, $p r=.02, n s$, or isometric handgrip tasks, $p r=-.023$, $n s$, after partialling baseline levels. In addition, between-task correlations for heart period, $p r=.27, p=.08$, and RSA, $p r=.31$, $p<.05$, were modest, whereas the between-task correlation for PEP, $p r=.20, n s$, did not reach statistical significance after partialling basal levels.

\section{Gastric Myoelectrical Activity}

The upper and lower panels of Figure 5 show that both speech preparation and the isometric handgrip task prompted a decrease in 3-cpm gastric myoelectrical activity and an increase in gastric tachyarrhythmia. A MANOVA, which included the percentages of 3-cpm activity and gastric tachyarrhythmia in the same model, revealed a main effect of Period, Pillai-Bartlett trace $=.194$, $F(4,49)=2.96, p=.03$, suggesting that the tasks affected gastric myoelectrical activity. Neither the main effect of Gender, nor the Gender $\times$ Period interaction reached statistical significance, $F s<1$. Repeated measures ANOVAs on the percentages of 3-cpm activity and gastric tachyarrhythmia revealed significant main effects of Period on both variables (percentage of 3-cpm activity: $F[2,104]=6.18, p=.003, \epsilon=.98$; percentage of gastric tachyarrhythmia: $F[2,104]=5.66, p=.005, \epsilon=.98)$. Post hoc comparisons of 3-cpm activity revealed differences between baseline and speech preparation, $t(56)=2.90, p=.005$, and between baseline and handgrip, $t(53)=2.90, p=.005$, but not between handgrip and speech preparation, $t<1$. Similarly, post hoc comparisons of gastric tachyarrhythmia revealed significant differences between baseline and speech preparation, $t(56)=2.71, p=$ .009 , and between baseline and handgrip, $t(53)=2.91, p=.005$, but not between handgrip and speech preparation, $t<1$. These 

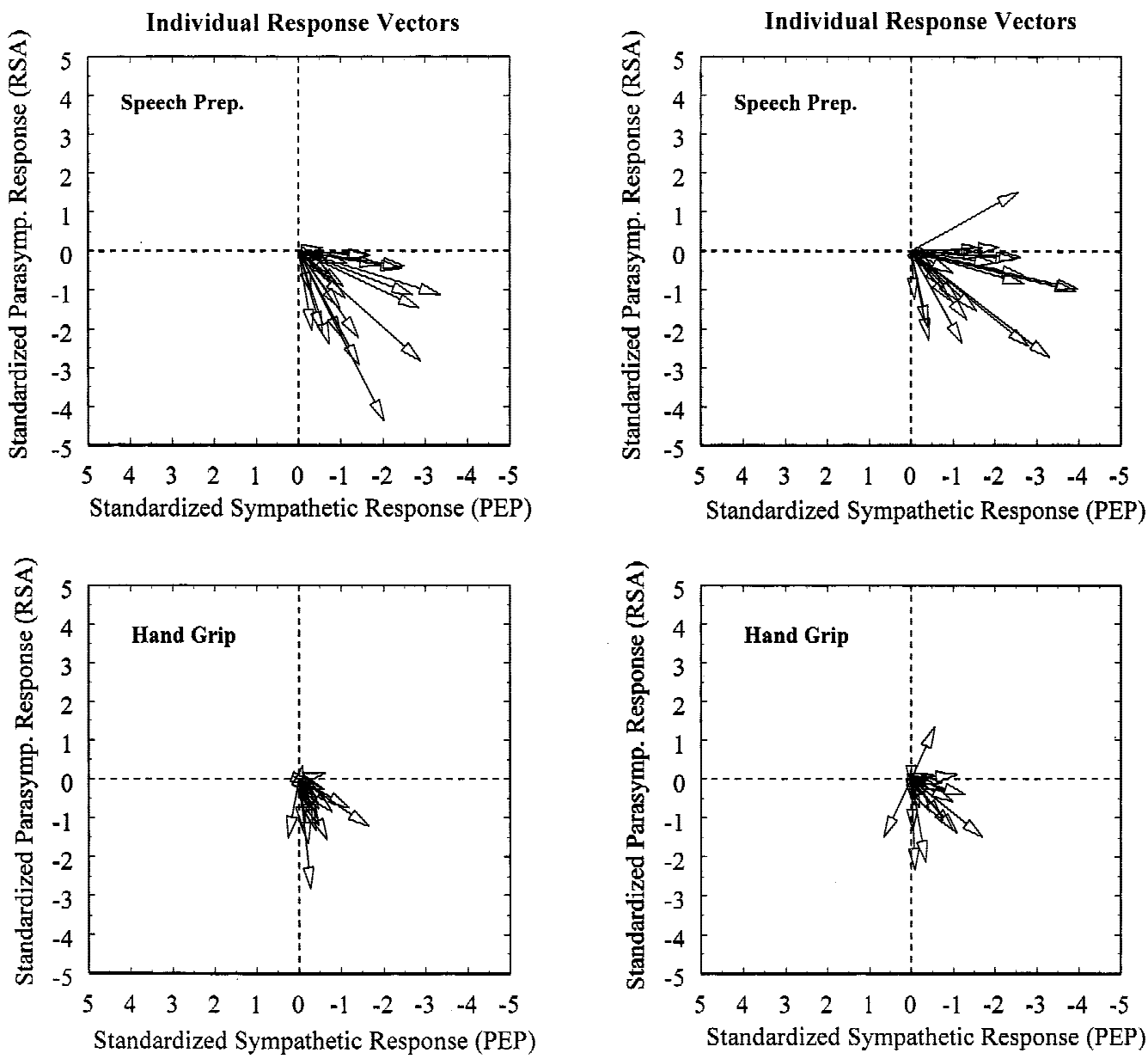

Figure 4. Individual autonomic response vectors for 43 participants. Data from the same randomly chosen subset of 22 participants are presented in the top and bottom panels on the left; the remaining participants are presented in the right panels. As illustrated in these panels, notable individual differences in autonomic response magnitude and autonomic mode of cardiac control are apparent both within and between tasks. Note that the sign of the values along the abscissa is reversed as in Figure 3, indicating that more negative values correspond to greater decreases in PEP.

data demonstrate that both tasks decreased normal 3-cpm gastric myoelectrical activity and increased gastric tachyarrhythmia.

Within the speech preparation task, no statistically significant partial correlations were observed between EGG activity and PEP (percentage of 3-cpm activity: $p r=.07, n s$; percentage of tachyarrhythmia: $p r=.08, n s$ ) or RSA (percentage of 3-cpm activity: $p r=.20, n s$; percentage of tachyarrhythmia: $p r=-.08, n s)$ after baseline levels of these variables were partialled. Similarly, EGG activity elicited by the isometric handgrip task did not correlate with PEP (percentage of 3-cpm activity: $p r=-.12, n s$; percentage of tachyarrhythmia: $p r=.19, n s$ ) or RSA (percentage of 3-cpm activity: $p r=.07, n s$; percentage of tachyarrhythmia: $p r=-.06$, $n s)$ after partialling basal levels. These data suggested that gastric myoelectrical reactivity to the laboratory stressors did not correlate with concurrent estimates of autonomic cardiac reactivity.

\section{Nausea Profile Scores}

Table 1 displays NP scores associated with each experimental period. Total NP scores differed significantly between the tasks, Pillai-Bartlett trace $=.680, F(2,54)=57.37, p<.001$. Neither the main effect of Gender, $F(1,55)=2.25$, nor the Gender $\times$ Period interaction, $F(2,54)=2.75$, reached statistical significance. Compared to the vanilla baseline task, total NP scores were greater during speech preparation, $t(56)=9.29, p<.001$, and the isometric handgrip task, $t(56)=8.49, p<.001$. Also, total NP scores were greater during speech preparation compared to the isometric handgrip task, $t(58)=3.11, p<.005$.

Although scores from the somatic subscale of the NP differed across task periods, the main effect of Period, Pillai-Bartlett trace $=$ $.624, F(2,54)=44.72, p<.001$, was modified by a Gender $\times$ Period interaction, $F(2,54)=3.17, p=.05$. The main effect of Gender, however, did not reach statistical significance, $F(1,55)=$ 2.12. Compared to males, females reported a higher level of somatic symptoms during speech preparation (males: $M=9.34$, $S E=2.06$; females: $M=17.51, S E=2.85), t(57)=2.26, p=.03$. Males and females did not differ in somatic symptom reports during the baseline period (males: $M=4.80, S E=1.14$; females: $M=9.25, S E=1.48$ ) or during isometric handgrip (males: $M=$ 24.31, $S E=3.46$; females: $M=21.33, S E=2.78$ ). Thus, although both speech preparation and the isometric handgrip task elicited an increase in somatic NP scores, females reported a greater level of somatic symptoms during speech preparation than males. 

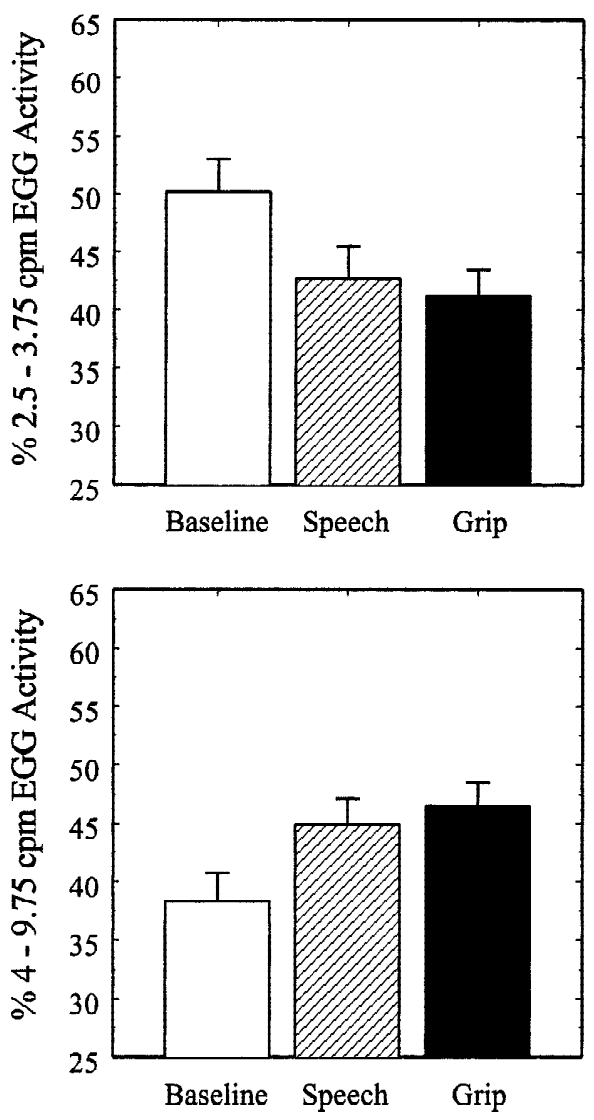

Figure 5. Percentages of 3-cpm gastric myoelectrical activity (upper panel) and gastric tachyarrhythmia (lower panel) during the baseline, speech preparation (Speech), and isometric handgrip (Grip) tasks. Error bars represent the standard error of the mean

Gastrointestinal subscale scores of the NP also differed across task periods, Pillai-Bartlett trace $=.180, F(2,54)=5.94, p=.005$. Neither the main effect of Gender, $F(1,55)=3.34$, nor the Gender $\times$ Period interaction, $F(2,54)=1.56$, reached statistical significance. Gastrointestinal subscale scores were greater during the speech preparation period compared to baseline, $t(56)=3.89, p<$ .001 , and the isometric handgrip task, $t(58)=3.71, p<.001$. In contrast, gastrointestinal scores reported during the isometric handgrip task did not differ from baseline, $t(56)=1.20$. These data show that only the speech preparation period prompted an increase in gastrointestinal symptoms.

Table 1. Mean Nausea Profile (NP) Scores by Period

\begin{tabular}{lrrrr}
\hline \hline & \multicolumn{4}{c}{ Nausea profile scores } \\
\cline { 2 - 5 } Period & \multicolumn{1}{c}{ Total } & \multicolumn{1}{c}{ Somatic } & \multicolumn{1}{c}{ GI } & \multicolumn{1}{c}{ NA } \\
\hline Baseline & $3.75(0.56)$ & $7.54(1.04)$ & $1.48(0.51)$ & $1.66(0.52)$ \\
Speech prep. & $17.53(2.16)$ & $14.18(1.94)$ & $8.32(2.03)$ & $29.03(3.01)$ \\
Handgrip & $11.46(1.57)$ & $22.54(2.15)$ & $2.86(1.32)$ & $8.54(1.80)$ \\
Drum rotation & $27.47(2.54)$ & $32.57(2.76)$ & $36.55(3.90)$ & $14.82(2.00)$
\end{tabular}

Note: $\mathrm{GI}=$ Gastrointestinal; NA $=$ Negative Affect. Scores represent the percentage of total points possible for each NP dimension. Values in parentheses represent standard errors of the mean.
Table 2. Hierarchical Regression Analysis Predicting Motion Sickness Scores from Preejection Period, Respiratory Sinus Arrhythmia, and Somatization

\begin{tabular}{lrrrrr}
\hline \hline Variable & \multicolumn{1}{c}{$\beta$} & $p r$ & \multicolumn{1}{c}{$s r$} & \multicolumn{1}{c}{$r$} & \multicolumn{1}{c}{$t$} \\
\hline Set 1 & & & & & \\
Baseline PEP & -.20 & -.20 & -.20 & -.19 & -1.28 \\
Baseline RSA & .10 & .10 & .10 & .08 & 0.66 \\
Set 2 & & & & & \\
Task PEP & .13 & .04 & .04 & -.18 & -0.31 \\
Task RSA & -.76 & -.37 & -.32 & -.15 & $-2.41^{*}$ \\
Somatization & .38 & .39 & .34 & .51 & $2.56^{*}$ \\
& & & & & \\
\hline \hline
\end{tabular}

Note: $\mathrm{PEP}=$ Preejection Period; RSA = Respiratory Sinus Arrhythmia. Set $1 R^{2}=.05$; Set $2 \Delta R^{2}=.32$.

$* p<.05$.

As expected, negative affect ratings differed across task periods, Pillai-Bartlett trace $=.711, F(2,54)=66.46, p<.001$. Compared to baseline, greater levels of negative affect were observed during the speech preparation, $t(56)=12.15, p<.001$, and isometric handgrip periods, $t(56)=4.88, p<.001$. In addition, greater levels of negative affect were reported during the speech preparation period compared to the isometric handgrip task, $t(58)=$ $9.22, p<.001$. Males and females did not differ in overall reports of negative affect, $F(1,55)=1.14$, nor did males and females differentially report affective symptoms during the tasks (Gender $\times$ Period interaction: $F[2,54]=1.58$ ).

Mean NP scores obtained following drum rotation are displayed in Table 1. Each drum rotation NP score differed significantly from corresponding NP scores observed during the baseline, speech preparation, and handgrip periods, all $p \mathrm{~s}<.005$. The mean maximum motion sickness score obtained during drum rotation, as assessed by the PDI, was 12.47 ( $S E=1.18$ ) out of a possible total of 64. No differences between males and females were observed for any of the drum rotation NP or PDI scores.

\section{Motion Sickness Prediction}

The results of the hierarchical regression analysis predicting motion sickness scores are presented in Table 2. In the first step, basal levels of PEP and RSA did not account for a significant proportion of the variance in motion sickness scores, $R^{2}=.05, F<1$. In contrast, the second set of variables, which included somatization scores along with average task estimates of RSA and PEP, accounted for a significant proportion of the remaining variance in motion sickness scores, $\Delta R^{2}=.32, F(3,37)=6.31, p=.001$. In this set, somatization and average task levels of RSA emerged as unique and significant predictors of motion sickness scores, after partialling baseline levels of autonomic cardiac activity. These data indicate that higher levels of somatization and greater taskinduced decreases in RSA predict an increased severity of motion sickness symptoms. ${ }^{3}$

${ }^{3}$ A multiple regression analysis was also conducted using somatization and average Task - Baseline change scores for RSA and PEP as predictors. The results of this analysis paralleled those of the hierarchical analysis: Somatization, $\beta=.42, p r=.43, s r=.38, r=.53, t=2.95, p=.005$, and $\Delta \mathrm{RSA}, \beta=-.30, p r=-.33, s r=-.28, r=-.46, t=-2.16, p=.04$, but not $\Delta$ PEP, $\beta=-.03, p r=-.03, s r=-.03, r=.04, t<1$, were found to uniquely predict motion sickness scores, $R^{2}=.36, p=.001$. 
Although no correlations were observed between somatization scores and basal, $r=.14, n s$, or task levels, $r=-.02, n s$, of RSA, somatization did correlate with task levels of RSA after partialling baseline RSA, $p r=-.36, p=.02$. This finding suggests that greater decreases in RSA to the laboratory stressors are related to higher levels of somatization.

Hierarchical regression analyses were also used to predict vection-induced changes in gastric tachyarrhythmia and 3-cpm gastric myoelectrical activity. None of the regression models containing somatization, PEP, or RSA, however, reached statistical significance, all $F \mathrm{~s}<1$.

\section{Discussion}

In the present study, both the speech preparation and isometric handgrip tasks elicited an overall reciprocal increase in sympathetic and a decrease in parasympathetic cardiac activity. As expected, these patterns of autonomic response were observed in conjunction with a decrease in 3-cpm gastric myoelectrical activity and an increase in gastric tachyarrhythmia. These findings are consistent with prior motion sickness research showing that similar patterns of gastric myoelectrical reactivity are observed in conjunction with a reciprocal pattern of autonomic response (Hasler et al., 1995; Hu et al., 1991; Koch, Stern, Vasey, Seaton et al., 1990; Money et al., 1996; Uijtdehaage et al., 1992). We do not recommend, however, that inferences regarding gastric autonomic activity be made from PEP or RSA. Indeed, no correlations between these estimates of autonomic cardiac activity and gastric myoelectrical activity were observed within the speech preparation or handgrip tasks. Moreover, whereas the autonomic origins of RSA and PEP have been well documented (e.g., Berntson et al., 1993a; Cacioppo et al., 1994), the autonomic contributions to gastric myoelectrical activity in humans are less clear.

Another goal of the present study was to evaluate the effects of these laboratory stressors on subjective reports of somatic, gastrointestinal, and emotional symptoms related to nausea. Several studies have shown that decreased normal gastric myoelectrical activity and increased gastric tachyarrhythmia are associated with the experience of nausea under a variety of clinical and nonclinical conditions (for reviews, see Koch \& Stern, 1996, and Stern et al., 2000). In the present study, the speech preparation task prompted a decrease in 3-cpm activity, an increase in gastric tachyarrhythmia, and reports of somatic, emotional, and gastrointestinal symptoms. In contrast, the isometric handgrip task elicited a similar decrease in 3-cpm activity and increase in gastric tachyarrhythmia; however, participants reported somatic and emotional, but not gastrointestinal, symptoms during this task. Thus, although mild subjective symptoms related to the experience of nausea were reported during speech preparation, some dissociation between reports of nausea and gastric myoelectrical activity was observed during the isometric handgrip task. This dissociation may be due to differences in stimulus quality between the two stressors; however, future work is needed to explore this possibility.

The extent to which stressor-induced changes in autonomic cardiac activity and somatization scores predict responses to a nauseogenic stimulus was also evaluated. The results indicated that task-induced decreases in RSA to the stressors predicted increased reports of motion sickness during illusory self-motion. These results complement prior findings (Uijtdehaage et al., 1992) showing that increased cardiac vagal activity following a meal predicts lower reports of motion sickness. Further, the results of the present study also indicate that prior sympathetic cardiac reactivity to laboratory stressors does not predict motion sickness susceptibility. These results contrast with a prior suggestion by Cowings et al. (1986) that sympathetic reactivity is an important predictor of future motion sickness symptomatology. In that study, Cowings et al. relied upon increases in heart rate during the early minutes of exposure to a rotating chair as markers of sympathetic activation; however, those observed changes in heart rate may have arisen from coupled, reciprocal, or independent changes in the activity of the two autonomic branches (Berntson et al., 1991, 1993a).

In addition to autonomic predictors, personality traits, such as neuroticism, have been used to predict motion sickness susceptibility (e.g., Collins \& Lentz, 1977; Reason \& Brand, 1975). The results of these studies, however, were often mixed and no studies had examined the relationship between somatization and motion sickness susceptibility. In the present study, the hypothesis that higher levels of somatization would predict symptoms of motion sickness upon exposure to a rotating optokinetic drum was supported. These results indicate that reports of motion sickness appear to be related to an individual's tendency to report distress from somatic symptoms across a number of contexts. Further, increased somatization was correlated modestly with task-induced decreases in RSA; however, we are unaware of previous studies reporting a similar finding.

Although somatization scores and task-induced changes in RSA predicted motion sickness symptoms, none of these variables predicted vection-induced changes in gastric myoelectrical activity. There are at least two possible explanations for this pattern of results. First, changes in gastric myoelectrical activity, specifically increases in gastric tachyarrhythmia, are not invariant markers of the experience of nausea. That is, prior work has shown that the expression of gastric tachyarrhythmia may be dissociated from the report of nausea (e.g., Levine, Chillas, Stern, \& Knox, 2000), as was observed in the isometric handgrip task of the current study. Second, these findings may reflect a relatively weak relationship of RSA and PEP to gastric myoelectrical activity. Indeed, neither PEP nor RSA correlated with EGG activity during the speech preparation or isometric handgrip tasks. It is noteworthy that Uijtdehaage et al. (1992) also failed to observe correlations between RSA and gastric myoelectrical activity in a fasted group of participants; however, Uijtdehaage et al. did report moderate correlations in those participants who were fed a small breakfast. In the present study, all participants were fasted for at least $3 \mathrm{hr}$ prior to experimentation. Taken together, these findings suggest that autonomic activation of the gut during a fed state may correspond with changes in autonomic drive to the heart, which could contribute to correlated patterns of gastric myoelectrical and autonomic cardiac activity within a given experiment.

Several lines of future inquiry are encouraged by the present study. First, the use of ambulatory EGG monitoring may help to determine whether the gastric myoelectrical responses observed in the present study are also elicited by stressors encountered in daily life. Second, to evaluate the generality of somatization and vagal reactivity as predictors of motion sickness susceptibility, future investigations should evaluate whether these variables predict symptomatology elicited by other nauseogenic contexts. Finally, stress reactivity is thought to play an important role in the development, maintenance, and exacerbation of symptoms related to functional gastrointestinal disorders (Mayer, 1999); therefore, it may prove useful to examine the relationship between a current or future clinical outcome and gastric myoelectrical reactivity to-or recovery from-laboratory stressors that are similar to those employed here. 


\section{REFERENCES}

Bassett-Frey, M. A., Doerr, B. M., \& Miles, D. S. (1982). Transthoracic impedance: Differences between men and women with implications for impedance cardiography. Aviation, Space, and Environmental Medicine, 12, 1190-1192.

Beaumont, W. (1833). Experiments and observations on the gastric juice and the physiology of digestion. New York: Dover.

Bennett, E. J., Piesse, C., Palmer, K., Badcock, C. A., Tennant, C. C., \& Kellow, J. E. (1997). Functional gastrointestinal disorders: Psychological, social, and somatic features. Gut, 42, 414-420.

Bennett, E. J., Tennant, C. C., Piesse, C., Badcock, C. A., \& Kellow, J. E. (1998). Level of chronic life stress predicts clinical outcome in irritable bowel syndrome. Gut, 43, 256-261.

Berntson, G. G., Bigger, J. T., Eckberg, D. L., Grossman, P., Kaufmann, P. G., Malik, M., Nagaraja, H. N., Porges, S. W., Saul, J. P., Stone, P. H., \& van der Molen, M. W. (1997). Heart rate variability: Origins, methods, and interpretive caveats. Psychophysiology, 34, 623-648.

Berntson, G. G., Cacioppo, J. T., Binkley, P. F., Uchino, B. N., Quigley, K. S., \& Fieldstone, A. (1994). Autonomic cardiac control III: Psychological stress and cardiac response in autonomic space as revealed by pharmacological blockades. Psychophysiology, 31, 599-608.

Berntson, G. G., Cacioppo, J. T., \& Fieldstone, A. (1996). Illusions, arithmetic, and the bi-directional modulation of vagal control of the heart. Biological Psychology, 44, 1-17.

Berntson, G. G., Cacioppo, J. T., \& Quigley, K. S. (1991). Autonomic determinism: The modes of autonomic control, the doctrine of autonomic space and the laws of autonomic constraint. Psychological Review, 98, 459-487.

Berntson, G. G., Cacioppo, J. T., \& Quigley, K. S. (1993a). Respiratory sinus arrhythmia: Autonomic origins, physiological mechanisms, and psychophysiological implications. Psychophysiology, 30, 183-196.

Berntson, G. G., Cacioppo, J. T., \& Quigley, K. S. (1993b). Cardiac psychophysiology and autonomic space in humans: Empirical perspectives and conceptual implications. Psychological Bulletin, 114, 296-322.

Berntson, G. G., Cacioppo, J. T., \& Quigley, K. S. (1995). The metrics of cardiac chronotropism: Biometric perspectives. Psychophysiology, 32, 162-171.

Berntson, G. G., Quigley, K. S., Jang, J. F., \& Boyson, S. T. (1990). An approach to artifact identification: Application to heart period data. Psychophysiology, 27, 586-598.

Cacioppo, J. T., Berntson, G. G., Binkley, P. F., Quigley, K. S., Uchino, B. N., \& Fieldstone, A. (1994). Autonomic cardiac control II: Noninvasive indices and basal response as revealed by autonomic blockades. Psychophysiology, 31, 586-598.

Camilleri, M., Malagelada, J.-R., Kao, P. C., \& Zinsmeister, A. R. (1984). Effect of somatovisceral reflexes and selective dermatomal stimulation on postcibal antral pressure activity. American Journal of Physiology, 247, G703-G708.

Camilleri, M., Malagelada, J-R., Kao, P. C., \& Zinsmeister, A. R. (1986). Gastric and autonomic responses to stress in functional dyspepsia. Digestive Diseases and Sciences, 31, 1169-1177.

Cohen, J., \& Cohen, P. (1983). Applied multiple regression/correlation for the behavioral sciences. Hillsdale, NJ: Erlbaum.

Collins, W. E., \& Lentz, M. (1977). Some psychological correlates of motion sickness susceptibility. Aviation, Space, and Environmental Medicine, 48, 587-594.

Costa, P. T., \& McCrae, R. R. (1987). Neuroticism, somatic complaints, and disease: Is the bark worse than the bite? Journal of Personality, 55, 299-316.

Cowings, P. S., Suter, S., Toscano, W. B., Kamiya, J., \& Naifeh, K. (1986). General autonomic components of motion sickness. Psychophysiology, $23,542-551$.

Derogatis, L. R. (1977). SCL-90: Administration, scoring, and procedure manual-I. Baltimore, MD: Johns Hopkins.

Derogatis, L. R., Rickels, K., \& Rock, A. F. (1976). The SCL-90 and the MMPI: A step in the validation of a new self-report scale. British Journal of Psychiatry, 128, 280-289.

Ernst, J. M., Litvack, D. A., Lozano, D. L., Cacioppo, J. T., \& Berntson, G. G. (1999). Impedance pneumography: Noise as signal in impedance cardiography. Psychophysiology, 38, 333-338.

Geldof, H., van der Schee, E. J., Smout, A. J. P. M., van de Merwe, J. P., van Blankenstein, M., \& Grashuis, J. L. (1989). Myoelectrical activity in gastric ulcer patients: An electrogastrographic study. Gastrointestinal Motility, 1, 122-130
Graybiel, A., Wood, C. D., Miller, E. F., \& Cramer, D. B. (1968). Diagnostic criteria for grading the severity of acute motion sickness. Aerospace Medicine, 39, 453-455.

Greenhouse, S., \& Geisser, S. (1959). On methods in the analysis of profile data. Psychometrika, 24, 95-112.

Grossman, P., Karemaker, J., \& Wieling, W. (1991). Prediction of tonic parasympathetic cardiac control using respiratory sinus arrhythmia: The need for respiratory control. Psychophysiology, 28, 201-216.

Hasler, W. L., Kim, M. S., Chey, W. D., Stevenson, V., Stein, B., \& Owyang, C. (1995). Central cholinergic and alpha-adrenergic mediation of gastric slow wave dysrhythmias evoked during motion sickness. American Journal of Physiology, 268, G539-G549.

Hochstrasser, B., \& Angst, J. (1996). The Zurich Study XXII: Epidemiology of gastrointestinal complaints and comorbidity with anxiety and depression. European Archives of Psychiatry and Clinical Neuroscience, 246, 261-272

Holtman, G., Singer, M. V., Kriebel, R., Stäcker, K. H., \& Goebell, H. (1989). Differential effects of acute mental stress on interdigestive secretion of gastric acid, pancreatic enzymes, and gastroduodenal motility. Digestive Diseases and Sciences, 34, 1701-1707.

Hu, S., Grant, W. F., Stern, R. M., \& Koch, K. L. (1991). Motion sickness severity and physiological correlates during repeated exposures to a rotating optokinetic drum. Aviation, Space, and Environmental Medicine, 62, 308-314.

Jennings, J. R., Kamarck, T., Stewart, C., Eddy, M., \& Johnson, P. (1992). Alternate cardiovascular baseline assessment techniques: Vanilla or resting baseline. Psychophysiology, 29, 742-750.

Kennedy, R. S., Dunlap, W. P., \& Fowlkes, J. E. (1990). Prediction of motion sickness susceptibility. In G. H. Crampton (Ed.), Motion and space sickness. Boca Raton, FL: CRC Press.

Kino, M., Lance, V. Q., Shahamatpour, A., \& Spodick, D. H. (1975). Effects of age on responses to isometric exercise. American Heart Journal, 90, 575-581.

Koch, K. L. (1993). Stomach. In M. M. Schuster (Ed.), Atlas of gastrointestinal motility in health and disease (pp. 158-176). Baltimore: Williams and Wilkins.

Koch, K. L. (1998). Clinical approaches to unexplained nausea and vomiting. Advances in Gastroenterology, Hepatology, \& Clinical Nutrition, 3, 163-178.

Koch, K. L., \& Stern, R. M. (1996). Functional disorders of the stomach. Seminars in Gastrointestinal Disease, 7, 185-195.

Koch, K. L., Stern, R. M., Vasey, M. W., \& Dwyer, A. (1990). Gastric dysrhythmias and nausea of pregnancy. Digestive Diseases and Sciences, 35, 961-968.

Koch, K. L., Stern, R. M., Vasey, M. W., Seaton, J. F., Demers, L. M., \& Harrison, T. S. (1990). Neuroendocrine and gastric myoelectrical responses to illusory self-motion in humans. American Journal of Physiology, 258, E304-E310.

Kohl, R. L., \& Homick, J. L. (1983). Motion sickness: A modulatory role for the central cholinergic nervous system. Neuroscience \& Biobehavioral Reviews, 7, 73-85.

Levine, M. E., Chillas, J. C., Stern, R. M., \& Knox, G. W. (2000). The effects of serotonin (5-HT3) receptor antagonists on gastric tachyarrhythmia and the symptoms of motion sickness. Aviation, Space, and Environmental Medicine, 71, 1111-1114.

Lewis, R. P., Leighton, R. F., Forester, W. F \& Weissler, A. M. (1974). Systolic time intervals. In A. M. Weissler (Ed.), Non-invasive cardiology (pp. 301-368). New York: Grune and Straton.

Liberski, S. M., Koch, K. L., Atnip, R. G., \& Stern, R. M. (1990). Ischemic gastroparesis: Resolution of nausea, vomiting, and gastroparesis after mesenteric artery revascularization. Gastroenterology, 99, 252-257.

Lydiard, R. B., Greenwald, S., Weissman, M. M., Johnson, J., Drossman, D. A., \& Ballenger, J. C. (1994). Panic disorder and gastrointestinal symptoms: Findings from the NIMH Epidemiologic Catchment Area Project. American Journal of Psychiatry, 151, 64-70.

Marten, P. A., Brown, T. A., Barlow, D. H., Borkovec, T. D., Shear, M. K., \& Lydiard, R. B. (1993). Evaluation of the ratings comprising the associated symptom criterion of the DSM-III-R generalized anxiety disorder. The Journal of Nervous and Mental Disease, 181, $676-682$.

Mayer, E. A. (1999). Emerging disease model for functional gastrointestinal disorders. American Journal of Medicine, 107, 12S-19S.

Miller, J. C., Sharkey, T. J., Graham, G. A., \& McCauley, M. E. (1993). 
Autonomic physiological data associated with simulator discomfort. Aviation, Space, and Environmental Medicine, 64, 813-819.

Money, K. E., Lackner, J. R., \& Cheung, R. S. K. (1996). The autonomic nervous system and motion sickness. In B. J. Yates \& A. D. Miller (Eds.), Vestibular autonomic regulation. New York: CRC Press.

Muth, E. R., Koch, K. L., Stern, R. M., \& Thayer, J. F. (1999). Effect of autonomic nervous system manipulations on gastric myoelectrical activity and emotional responses in healthy human subjects. Psychosomatic Medicine, 61, 297-303.

Muth, E. R., Stern, R. M., \& Koch, K. L. (1998). A psychophysiological paradigm for the study of nausea: A rotating optokinetic drum, the electrogastrogram and the nausea profile. Journal of Psychophysiology, $12,56-63$.

Muth, E. R., Stern, R. M., Thayer, J. F., \& Koch, K. L. (1996). Assessment of the multiple dimensions of nausea: The Nausea Profile (NP). Journal of Psychosomatic Research, 40, 511-520.

Parker, D. M. (1971). A psychophysiological test for motion sickness susceptibility. Journal of General Psychology, 85, 87-92.

Pollack, M. H., \& Obrist, P. A. (1988). Effects of autonomic blockade on heart rate responses to reaction time and sustained handgrip tasks. Psychophysiology, 25, 689-695.

Porges, S. W., \& Bohrer, R. E. (1990). The analysis of periodic processes in psychophysiological research. In J. T. Cacioppo \& L. G. Tassinary (Eds.), Principles of Psychophysiology: Physical, Social, and Inferential Elements (pp. 708-753). Cambridge: Cambridge University Press.

Reason, J. T., \& Brand., J. J. (1975). Motion sickness. London: Academic Press.

Riezzo, G., Porcelli, P., Guerra, V., \& Giorgio, I. (1996). Effects of different psychophysiological stressors on the cutaneous electrogastrogram in healthy subjects. Archives of Physiology and Biochemistry, 104, 282-286.

Saab, P., Matthews, K., Stoney, C., \& McDonald, R. (1989). Pre-menopausal and post-menopausal women differ in their cardiovascular and neuroendocrine response to behavioral stressors. Psychophysiology, 29, 734-741.

Sherwood, A., Allen, M. T., Fahrenberg, J., Kelsey, R. M., Lovallo, W. R., \& van Doornen, L. J. P. (1990). Methodological guidelines for impedance cardiography. Psychophysiology, 27, 1-23.

Stern, R. M. (1983). Responsiveness of the stomach to environmental events. In R. Hölzl \& W. E. Whitehead (Eds.), Psychophysiology of the gastrointestinal tract. Experimental and clinical applications. New York: Plenum Press.

Stern, R. M., \& Higgins, D. (1969). Perceived somatic reactions to stress: Sex, age, and familial occurrence. Journal of Psychosomatic Research, $13,77-82$

Stern, R. M., \& Koch, K. L. (1996). Motion sickness and differential susceptibility. Current Directions in Psychological Science, 5, 115-120.

Stern, R. M., Koch, K. L., Leibowitz, H., Lindbald, I., Shupert, C., \& Stewart, W. R. (1985). Tachygastria and motion sickness. Aviation, Space, and Environmental Medicine, 56, 1074-1077.
Stern, R. M., Koch, K. L., \& Muth, M. W. (2000). The gastrointestinal system. In J. T. Cacioppo, L. G. Tassinary, \& G. G. Berntson (Eds.), Handbook of psychophysiology, 2nd ed. (pp. 294-314). Cambridge: Cambridge University Press.

Stern, R. M., Koch, K. L., Stewart, W. R., \& Lindbald, I. M. (1987). Spectral analysis of tachygastria recorded during motion sickness. Gastroenterology, 92, 92-97.

Stern, R. M., Vasey, M. W., Hu, S., \& Koch, K. L. (1991). Effects of cold stress on gastric myoelectric activity. Journal of Gastrointestinal Motility, $3,225-228$.

Stewart, W. R. (1987). Stress-induced alterations in gastric myoelectric activity as measured with the electrogastrogram. Unpublished doctoral dissertation, The Pennsylvania State University.

Thompson, D. G., Richelson, E., \& Malagelada, J.-R. (1982a). Perturbation of human gastroduodenal motility via the central nervous system. In M. Wienbeck (Ed.), Motility and the digestive tract (pp. 181-183). New York: Raven.

Thompson, D. G., Richelson, E., \& Malagelada, J.-R. (1982b). Perturbation of gastric emptying and duodenal motility through the central nervous system. Gastroenterology, 83, 1200-1206.

Thompson, D. G., Richelson, E., \& Malagelada, J.-R. (1983). Perturbation of gastrointestinal function by cold stress. Gut, 24, 277-283.

Uijtdehaage, S. H. J., Stern, R. M., \& Koch, K. L. (1992). Effects of eating on vection-induced motion sickness, cardiac vagal tone, and gastric myoelectric activity. Psychophysiology, 29, 193-201.

Uijtdehaage, S. H. J., Stern, R. M., \& Koch, K. L. (1993). Effects of scopolamine on autonomic profiles underlying motion-sickness susceptibility. Aviation, Space, and Environmental Medicine, 64, 1-8.

Watson, D., \& Pennebaker, J. W. (1989). Health complaints, stress, and distress: Exploring the central role of negative affectivity. Psychological Review, 96, 234-254.

Whitehead, W. E. (1992). Behavioral medicine approaches to gastrointestinal disorders. Journal of Consulting and Clinical Psychology, 60, 605-612.

Whitehead, W. E. (1996). Psychological aspects of functional gastrointestinal disorders. Gastroenterology Clinics of North America, 25, 21-34.

Whitehead, W. E., Crowell, M. D., Robinson, J. C., Heller, B. R., \& Schuster, M. M. (1992). Effects of stressful life events on bowel symptoms: Subjects with irritable bowel syndrome compared to subjects without bowel dysfunction. Gut, 33, 825-830.

Wilding, J. M., \& Meddis, R. (1972). A note on personality correlates of motion sickness. British Journal of Psychology, 63, 619-620.

Wolf, S., \& Wolff, H. G. (1947). Human gastric function: An experimental analysis of a man and his stomach. Oxford, London: Oxford Press.

(Received August 21, 2000; ACCePted December 15, 2000) 